\title{
2D Flow in a Wall-bounded Porous Medium
}

\author{
PAULO J. S. A. Ferreira de Sousa ${ }^{1,2, a}$, ISABEL Malico ${ }^{1,2, b}$ and GÉRSON \\ Fernandes ${ }^{1, c}$ \\ ${ }^{1}$ University of Évora, Department of Physics, R. Romão Ramalho, 59, 7000-671 Évora, \\ Portugal \\ ${ }^{2}$ IDMEC/IST, Technical University of Lisbon, Department of Mechanical Engineering, Av. \\ Rovisco Pais, 1049-001 Lisbon, Portugal \\ aferreiradesousa@gmail.com, bimbm@uevora.pt, ${ }^{\mathrm{c}}$ gersonf@uevora.pt
}

Keywords: Immersed boundary method. Incompressible flow. Square cylinder. Porous media.

\begin{abstract}
A compact finite differences method is used to calculate two-dimensional viscous flows through complex geometries. The immersed boundaries are set through body forces that allow for the imposition of boundary conditions that coincide with the computational grid. Two different flow configurations are simulated. First, the flow through a row of cylinders with square cross-sections is calculated and used as a validation study. The computed average drag coefficient and Strouhal number are compared to data available in the literature, showing a good agreement between the results. The second flow configuration analyzed is the flow through a porous matrix composed of equal size staggered square cylinders. Flow visualization results are shown and various flow regimes identified. Different inlet boundary conditions are compared. The drag coefficient is larger when a uniform inlet velocity is prescribed and the variability between cylinders is lower.
\end{abstract}

